\title{
Ultrasound diagnosis of delayed-onset congenital diaphragmatic hernia associated with group B streptococcal infection*
}

\author{
D. Poenaru', J.-M. Laberge' ${ }^{1}$, S. Jéquier ${ }^{2}$, P. W. Blanchard ${ }^{3}$, and D. P. Doody ${ }^{1}$ \\ Departments of 'Pediatric Surgery, ${ }^{2}$ Pediatric Radiology, and ${ }^{3}$ Newborn Medicine, The Montreal Children's Hospital, and the \\ McGill University, Montreal Children's Hospital Research Institute, 2300 Tupper Street, Montreal, Quebec, Canada, H3H 1P3
}

\begin{abstract}
Delayed presentation of right-sided congenital diaphragmatic hernia in association with neonatal group B streptococcal pneumonia is a recently recognized entity. Its diagnosis is based on characteristic yet nonspecific chest X-ray findings, frequently supplemented by a variety of other imaging techniques. We describe two cases in which the diagnosis was reached by ultrasound examination. In the presence of nonresolving neonatal streptococcal pneumonia with the radiographic appearance of a right pleural effusion or suggestion of an elevated right hemidiaphragm, a congenital diaphragmatic hernia should be suspected. In this context, ultrasound can provide early proof of hepatic herniation before gas-containing bowel loops are seen on the cest X-ray.
\end{abstract}

Key words: Congenital diaphragmatic hernia Neonatal streptococcal pneumonia - Ultrasound

\section{Introduction}

The association between delayed presentation of right-sided congenital diaphragmatic hernia $(\mathrm{CDH})$ and neonatal group B streptococcal pneumonia was first described in 1977 by Kenny et al. [7], and over 20 cases have since been reported [1]. The difficulty in diagnosis caused by the concomitant lung infection and the buttressing effect of the liver have led to the use of various imaging methods, including peritoneography, fluoros-

\footnotetext{
* This is publication number 87018 of the McGill University Montreal Children's Hospital Research Institute, which supported, in part, this work.

Offprint requests to: J.-M. Laberge
}

copy, and radionuclear scanning $[1,9]$. We report two cases of delayed right diaphragmatic hernia associated with neonatal streptococcal infection successfully diagnosed by ultrasound (US).

\section{Case reports}

Case 1 . This $3.2-\mathrm{kg}$ female infant was born at 40 weeks' gestation with normal Apgar scores. Four hours later she developed respiratory distress and was transferred to our hospital. Initial chest X-ray (CXR) demonstrated a right lung infiltrate with a small pleural effusion in keeping with extensive right-sided pneumonia (Fig. 1 top). Blood and superficial cultures grew group B Streptococcus sp. With intravenous antibiotics the infant improved slowly over the next 4 weeks, but the right-sided basal consolidation remained. Her condition then suddenly deteriorated, with the CXR revealing extensive right hemithoracic opacification, right pleural effusion, and rounded air collections in the right base (Fig. 1 bottom). This was thought to be compatible with a cavitating pneumonic process. Thoracentesis produced $10 \mathrm{ml}$ serosanguinous fluid, and fluoroscopy for evaluation of the diaphragm was not diagnostic. A static US examination provided the diagnosis by demonstrating a partially absent right hemidiaphragm, liver in the right chest, and possible intestinal herniation (Fig. 2). At surgery the intrathoracic liver and bowel were reduced and the posterior diaphragmatic defect repaired. Right lung re-expansion occurred quickly and the patient was discharged on the 5th postoperative day.

Case 2. This 2-week-old baby boy was transferred to our center for persistent respiratory distress after a 14-day course of parenteral antibiotics for culture-proven group B streptococcal infection. He was born at 38 weeks' gestation with a birth weight of $3.3 \mathrm{~kg}$. Respiratory distress ensued a few hours after birth. Progressive deterioration required intubation starting on the 2nd postnatal day, and subsequent attempts at extubation on days 4,8 , and 14 failed. CXR initially showed bilateral lower-lobe and left perihilar consolidation, consistent with bronchopneumonia (Fig. 3 top). Prior to transfer, radiographs demonstrated increased opacification of the right lung and a mediastinal shift to the left. The right hemidiaphragm appeared elevated (Fig. 3 bottom). At this point, because of experience with the first case, a CDH was suspected. US examina- 

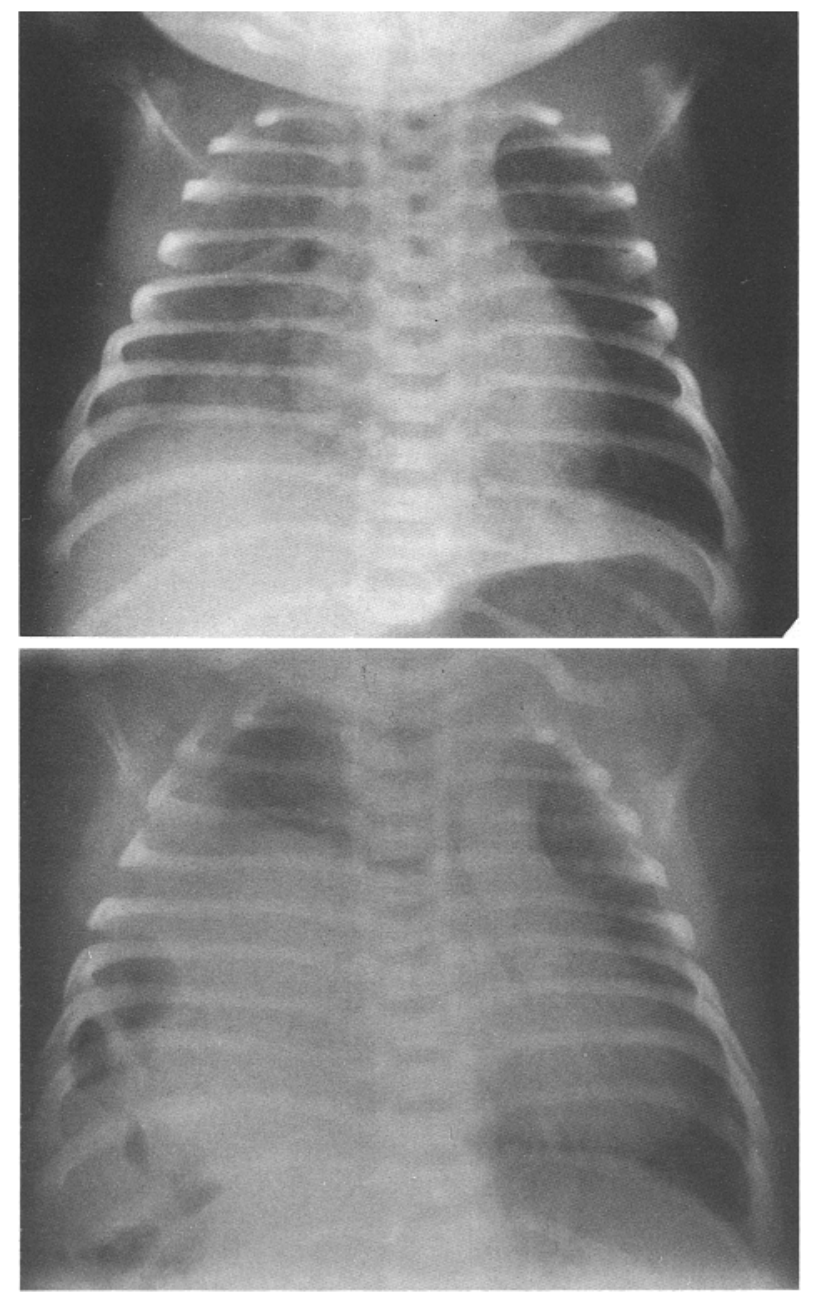

Fig. 1. Case 1. (top) CXR on the 1st day of life shows right lung consolidation with some loss of volume and a small pleural effusion. (Bottom) CXR at the age of 1 month now reveals opacification of right hemithorax, rounded air collections in the right base, and a right pleural effusion

tion demonstrated an intrathoracic liver with increased echogenicity of the right hepatic lobe compared to the left, absent right hemidiaphragm posteriorly, and only a small pleural effusion (Fig. 4). Real-time examination evidenced a paradoxical "rocking" motion of the liver, with the left lobe descending on inspiration while the right lobe ascended. Thoracotomy revealed a partial herniation of the liver through a posterolateral diaphragmatic defect, without intestinal herniation. After surgery, pulmonary function improved allowing extubation within $24 \mathrm{~h}$ and discharge from the hospital on the $6^{\text {th }}$ postoperative day.

\section{Discussion}

Ashcraft et al. [1] offer a review of the previous reports on delayed presentation of $\mathrm{CDH}$ following group B streptococcal pneumonia. They consider the pulmonary process secondary to inadequate diaphragmatic movement on the side of the her-

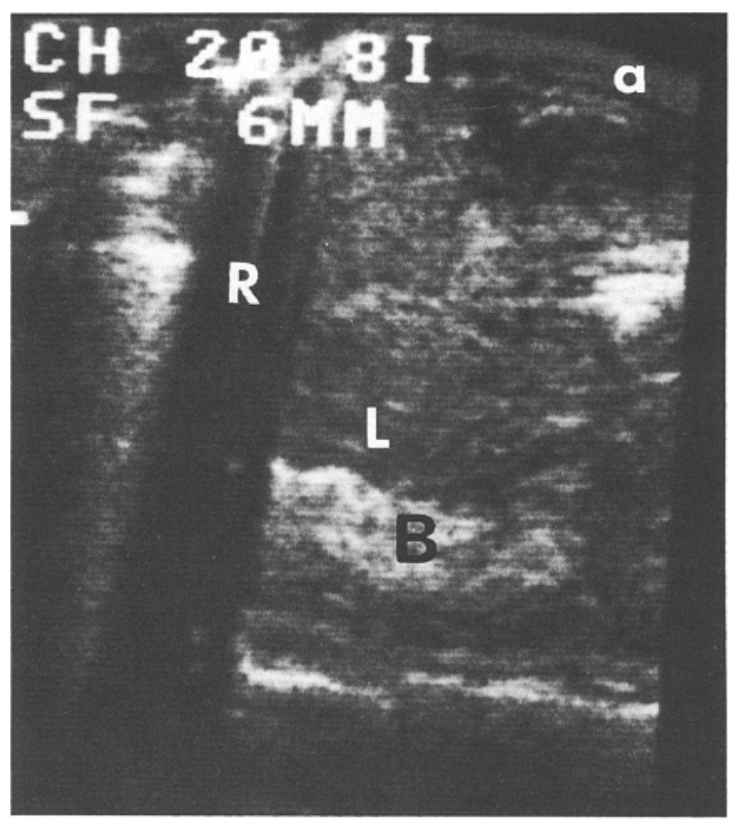

Fig. 2. Case 1. Sagittal US of the right chest, at 1 month of age. The liver is intrathoracic, the diaphragm cannot be outlined on this view, and an echogenic area posteriorly is compatible with herniated bowel ( $a$ anterior chest wall, $L$ liver, $R$ rib shadowing, $B$ bowel)

nia. However, deficient mechanical ventilation does not readily explain the rapid onset of the pneumonia and the positive blood and skin cultures. Banagale and Watters [2] see the infectious process as occurring independently of the anatomical defect, streptococcal sepsis being very common in newborns (1/330 live births [1]). They postulate that the parenchymal inflammatory changes, the increased intrathoracic pressures from mechanical ventilation, and the buttressing effect of the liver contribute to delaying actual herniation through the existent diaphragmatic defect. This latter theory may explain the usual clinical picture of initial improvement and extubation followed by recurrent respiratory distress. This deterioration probably coincides with delayed herniation through the congenital posterolateral defect.

The mainstay of diagnosis of delayed-onset $\mathrm{CDH}$ remains the CXR. The classical findings of initial right-lower-lobe density with normal-appearing diaphragm followed by right pleural effusion, elevated right hemidiaphragm, left mediastinal shift, and high hepatic flexure bowel gas have been previously described $[1,2,9]$ and were present in our two cases. However, these findings are not pathognomonic and the diagnosis is often made only at a later stage with the presence of in- 

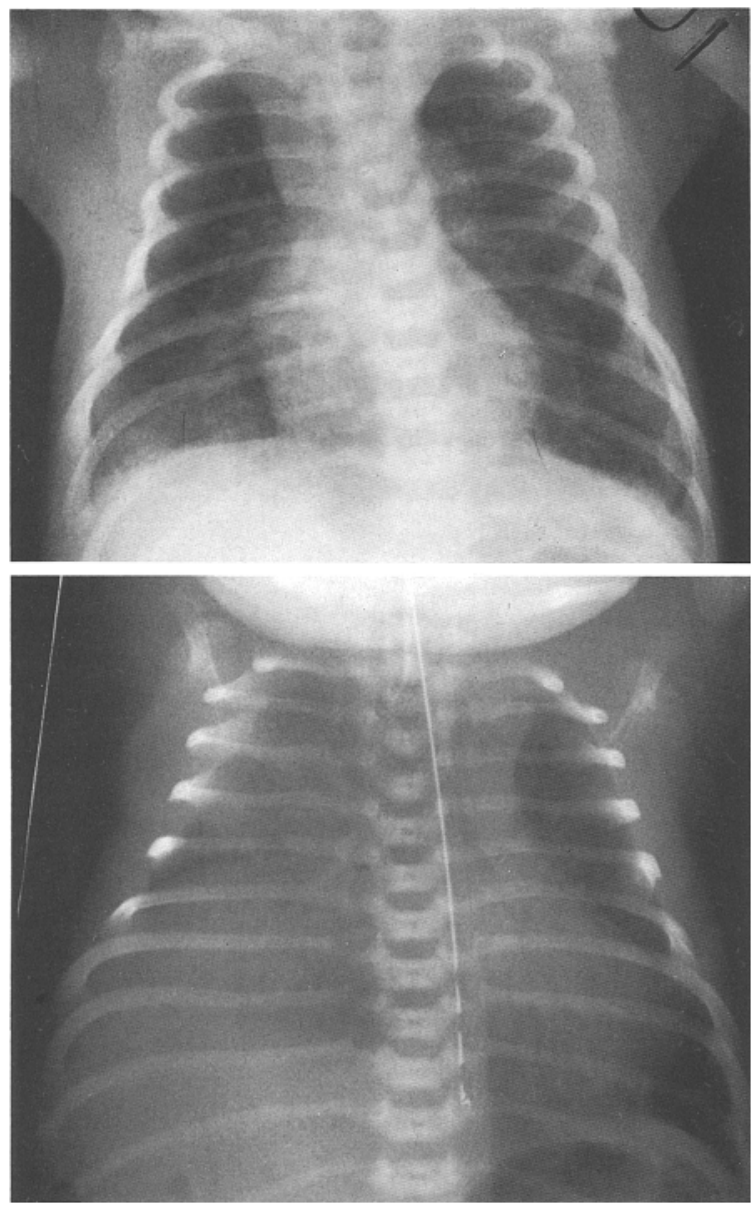

Fig. 3. Case 2. (top) CXR from the 1st day of life demonstrates patchy consolidation in both lower lobes and left perihilar region; the hemidiagphragms appear normal. (Bottom) CXR at 2 weeks of age. The right hemidiaphragm now appears elevated and the mediastinum is shifted towards the left. There is an increase in the consolidation of the right lung and a small pleural effusion

trathoracic bowel loops. To avoid this delay, a variety of adjunctive studies have been used. The new onset of pleural effusion in an unresolving pneumonia makes thoracentesis very tempting. This procedure is of little diagnostic use, as sterile serosanguinous fluid is almost alyways obtained and the risk of damaging an intrathoracic liver in this condition is real. Chilton et al. reported two cases, one with an intrahepatic chest tube at necropsy, the other with a hepatic laceration following thoracentesis [3]. Fluoroscopy can reveal diminished or paradoxical movement of the hemidiaphragm, but will not exclude eventration or diaphragmatic paralysis, both of which can mimic hernias $[1,2,9]$. Fluoroscopy is not useful in the mechanically ventilated patient, and in our first case it was falsely negative. Peritoneography has
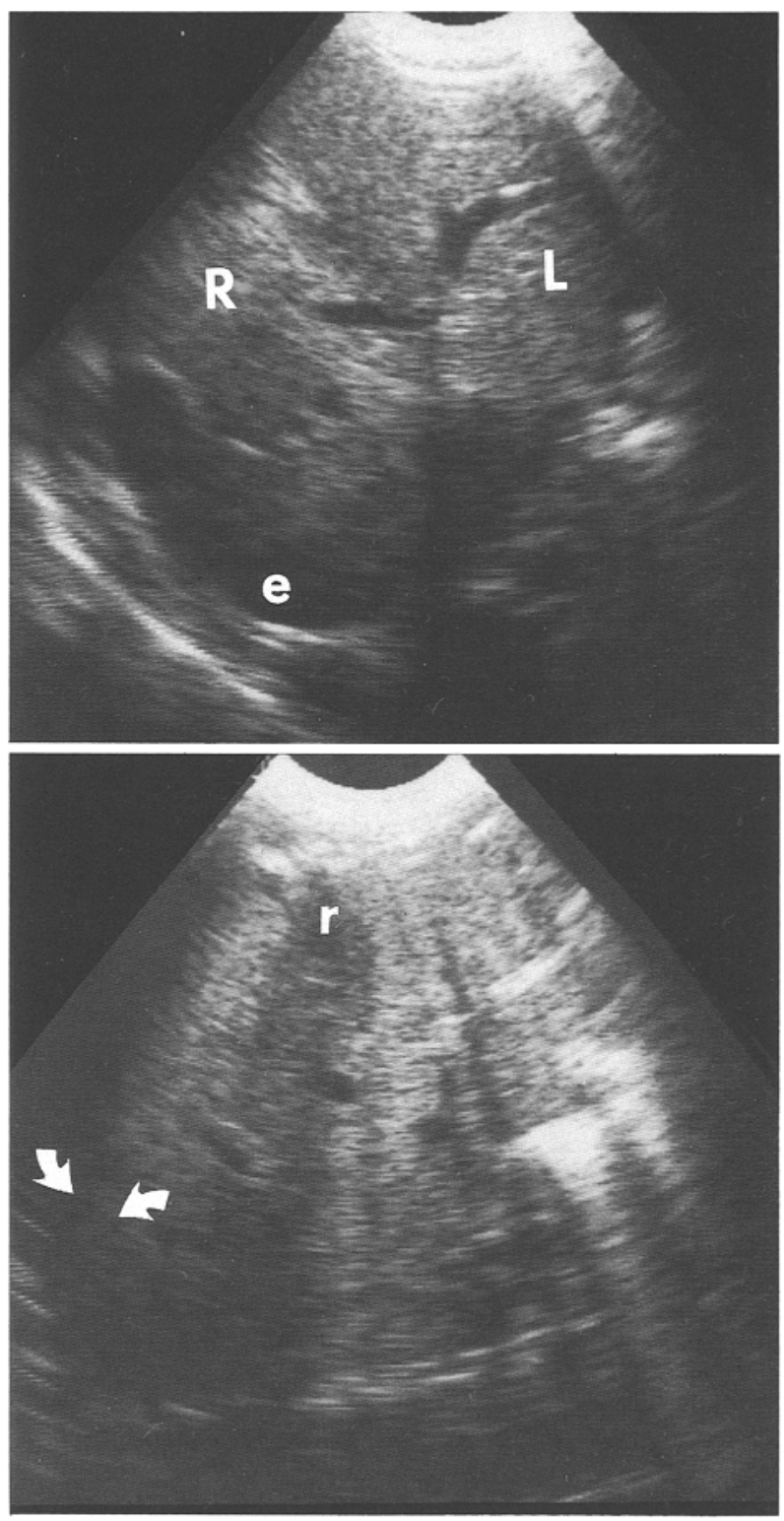

Fig. 4. Case 2. (top) Transverse US of the right upper quadrant at age 17 days. The right of the liver $(R)$ is more echogenic than the left $(L)$, there is a small right pleural effusion $(e)$, and the right hemidiaphragm is not visualized. (Bottom) Sagittal US of right chest and right upper quadrant on the same day: absence of a hemidiaphragm above the liver (should be between arrows), with elevation of the liver into the right hemithorax ( $r$ rib)

also been used, but if air is injected in this procedure rather than an iodinated contrast agent, hernial defects can be missed by air trapping under the anterior leaf of the diaphragm [6]. Liver scans and arteriography outline the position of the liver without specifically imaging the diaphragm-hence eventration will remain in the differential diagnosis [1]. Although computerized tomography (CT) 
was successfully applied to diagnose diaphragmatic hernias in asymptomatic adults [5], it is difficult to perform in the sick, ventilated newborn.

US was previously recommended for the diagnosis of late-onset $\mathrm{CDH}$, and Khan et al. [8] used it to diagnose a renal herniation through the canal of Bochdalek. We used US as early as 1980 (case 1) to diagnose diaphragmatic hernias, and in both of our cases this provided the final diagnosis. The US can usually demonstate an absent leaf of the diaphragm and/or intrathoracic liver, both findings being in favor of a right-sided diaphragmatic hernia. Moreover, as our second case showed, the US can reveal a partial hepatic herniation by showing differential echogenicity of the hepatic lobes. This is probably the result of venous congestion in the intrathoracic liver, which may also explain the pleural effusion. In addition, a mrocking " motion of the liver around an anteroposterior axis between the two lobes can be seen with respiration.

The absence of pulmonary hypoplasia in delayed CDH confers an excellent prognosis after surgical repair. An early diagnosis is essential in order to avoid the risk of intestinal incarceration with possible strangulation, as well as to relieve a perpetuating factor for the pneumonia and avoid the complications of prolonged intubation and oxygen toxicity. Daum and Roth [4] point out the delay in the diagnosis of a $\mathrm{CDH}$ from misdirected procedures such as tomography and thoracentesis; they also recommend ultrasound examination, with CT and liver scintigraphy as possible adjuncts. In this context, US offers a noninvasive, inexpensive, and rapid diagnostic tool. More experience will be needed to see to what extent US can assist in differentiating eventration of the diaphragm from congenital hernia. The sonographic detection of bowel loops in the right hemi- thorax and the different echogenicity of the right liver lobe as compared to the left are probably specific for herniation, but may not be present in all cases of $\mathrm{CDH}$. The clinical suspicion of a right-sided diaphragmatic hernia in patients with nonresolving streptococcal pneumonia together with diagnostic ultrasonography will lead to the earlier recognition and repair of this life-threatening congenital defect.

\section{References}

1. Ashcraft KW, Holder TM, Amoury RA, Hall FK, Rising WD, Hall RT, Sharp RJ (1983) Diagnosis and treatment of right Bochdalek hernia associated with group B streptococcus pneumonia and sepsis in the neonate. $\mathbf{J}$ Pediatr Surg 18: $480-485$

2. Banagale RC, Watters JH (1983) Delayed right-sided diaphragmatic hernia following group B streptococcal infection. Hum Pathol 14: 67-69

3. Chilton HW, Chang JHT, Jones MD, Brooks JG (1978) Right-sided congenital diaphragmatic hernia presenting as pleural effusions in the newborn. Arch Dis Child 53: $600-603$

4. Daum R, Roth H (1985) Delayed diagnosis in right-sided congenital diaphragmatic hernia. $Z$ Kinderchir 40 : 364-367

5. Gale ME (1985) Bochdalek hernia: prevalence and CT characteristics. Radiology 156:449-452

6. Graviss ER, Danis RK, Lewis JE, Beach PJ, Silberstein MJ, Brodeur AE (1980) Peritoneography-diagnosis of delayed-onset diaphragmatic hernias masquerading as pleural effusion. J Pediatr 97: 119-122

7. Kenny JD, Wagner ML, Harberg FF, Corbett AJ, Rudolph AJ (1977) Right-sided diaphragmatic hernia of delayed onset in the newborn. South Med J 70:373-374

8. Khan AN, Gould DA (1981) Sonographic diagnosis of right renal herniation through the canal of Bochdalek. J Clin Ultrasound 12: 237-238

9. McCarten KM, Rosenberg HK, Borden S, Mandell GA (1981) Delayed appearance of right diaphragmatic hernia associated with group B streptococcal infection in newborns. Radiology 139: 385-389

Accepted April 28, 1987 